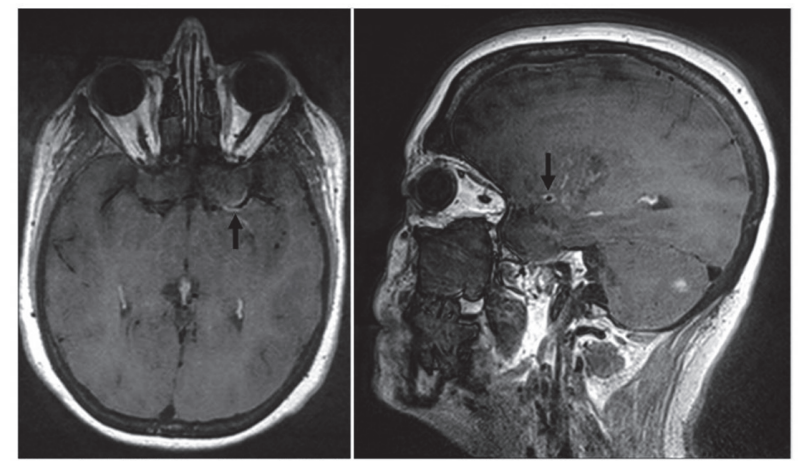

Abstract P-018 Figure 1 Axial (left) and Sagittal (right) post-contrast 3D T1 black blood sequence showing circumferential vessel wall enhancement of the left M1 segment in a patient who recently received mechanical thrombectomy with three passes of the stent retriever

gadolinium. Enhancement data variables from the MRI that were recorded included pattern (circumferential versus eccentric) and amount of maximal contrast enhancement based on region of interest differences in the region of highest T1 signal between post and pre-contrast T1 sequences. Following thrombectomy, the type and number of passes with each device was recorded.

Results 10 adult patients (6 F, Mean age of 65.7 years) were included in this study. Two received stent retriever with aspiration catheter, 7 received stent retriever only, and 1 received aspiration only. All 10 patients demonstrated circumferential enhancement in the region of retrieved clot, including the one patient who received aspiration only. Of the 9 patients that received the stent retriever, recanalization was achieved in 1 pass in 4, while in the other 5 patients, recanalization was achieved after multiple passes (range 2-4 passes). Dichotomizing the data between 1 and multiple passes, there was a difference in the amount of mean maximal enhancement of the involved vessel wall (single pass: 75, Std Dev 28.39 vs multiple passes: 110.6, Std Dev 51.75), although this did not reach statistical significance due to a small number of patients.

Conclusions All patients showed circumferential wall enhancement despite type of thrombectomy technique, suggesting that all techniques may be associated with a similar mechanism of endothelial wall injury. Moreover, those patients who required multiple passes of the stent retriever for recanalization demonstrated a higher mean maximal enhancement when compared to those patients who required just one pass. Further exploration with an increased number of patients is warranted.

Disclosures R. Mattay: None. C. Favilla: None. B. Pukenas: None. O. Choudhri: None. R. Hurst: None. S. Nabavizadeh: None.

\section{P-019 CLINICAL SIGNIFICANCE OF CONTRAST STAINING AFTER MECHANICAL THROMBECTOMY FOR ACUTE ISCHEMIC STROKE}

T Lazaro*, P Cotton, C English, K Katlowitz, O Tanweer, D Raper. Neurosurgery, Baylor College of Medicine, Houston, TX

\subsection{6/neurintsurg-2021-SNIS.55}

Background Acute ischemic stroke is a leading cause of mortality and morbidity throughout the world. Thrombolysis with intravenous tissue plasminogen activator (tPA) is often the first-line therapy after presentation. For select patients with large vessel occlusions (LVO), mechanical thrombectomy (MT) is the gold standard to achieve cerebral reperfusion, in which a variety of clot retriever devices and aspiration catheters are employed to remove the clot. After MT, a computed tomography (CT) scan of the head is often done shortly after to evaluate for stroke progression and hemorrhagic transformation; however, in some patients, contrast can remain within the parenchyma of the brain, referred to as contrast staining (CS). While an unclear radiologic finding, previous studies have suggested that it represents injury to small vessels branching from the occluded vessel during thrombectomy (Renu et al. Stroke. 2014). As a result, CS has been associated with a higher risk intracerebral hemorrhage $(\mathrm{ICH})$ after $\mathrm{MT}$.

Objective In this study, we look to (1) better characterize the clinical significance of post-MT CS in our cohort of acute ischemic stroke patients and (2) determine whether specific devices deployed at the time of thrombectomy are correlated with CS.

Methods We retrospectively reviewed patients from 2017-2020 who underwent MT. Included patients were those that presented within a LVO (i.e. clot within the internal carotid artery, proximal anterior cerebral artery, proximal middle cerebral artery, vertebral artery, or basilar artery) and underwent MT within 24 hours of symptom onset. Patients that had vessel occlusions related to prior surgical or endovascular procedures were excluded. Clinical outcomes were reported with the modified Rankin Scale (mRS).

Results Patients with CS were diagnosed with early ICH (diagnosed within 24 hours after MT) more often that patients without CS $(59 \%$ vs $18 \%$, respectively; p < 0.001$)$. The groups did not differ in late ICH (diagnosed after 24 hours). Patients with symptomatic ICH had higher $\mathrm{mRS}$ at discharge (median of 5 vs 3 , respectively; $p=0.02$ ). However, CS was not independently associated with worsened mRS after MT $(\mathrm{p}=0.47)$. In addition, larger diameter clot retriever devices and catheters were not significantly correlated with the presence of contrast staining or development of symptomatic ICH.

Conclusion Contrast staining is associated with the development of ICH, particularly in the early period after MT and worse clinical outcomes. While patients with $\mathrm{ICH}$ had worse functional outcomes at discharge, contrast staining itself was not associated with worse outcomes. Lasty, larger diameter clot retriever devices and catheters were not significantly correlated with the presence of contrast staining.

Disclosures T. Lazaro: None. P. Cotton: None. C. English: None. K. Katlowitz: None. O. Tanweer: None. D. Raper: None.

\section{P-020 PROCESS IMPROVEMENT WITH PRE-TRANFSER INTUBATION FOR PATIENTS UNDERGOING MECHANICAL THROMBECTOMY; NOT JUST FOR THE COVID PANDEMIC}

\footnotetext{
${ }^{1,2} \mathrm{M}_{\text {Stiefel }}{ }^{*},{ }^{1,2} \mathrm{~J} \mathrm{Strauss,}^{3} \mathrm{~J}$ Shapiro, ${ }^{3} \mathrm{~S}$ Solomon, ${ }^{2} \mathrm{C}$ Lytle, ${ }^{2} \mathrm{~T}$ Esdale, ${ }^{1,2} \mathrm{~A}$ Schuette. ${ }^{1}$ Cerebrovascular and Endovascular Neurosurgery, Piedmont Healthcare, Atlanta, GA; ${ }^{2}$ Piedmont Stroke Center, Atlanta, GA; ${ }^{3}$ Anesthesia, Piedmont Healthcare, Atlanta, GA
}

10.1136/neurintsurg-2021-SNIS.56 